DOI: https://doi.org/10.15517/rce.v36i2.35709

\title{
TENDENCIAS Y TIPO DE RAZONAMIENTO ÉTICO ENTRE GERENTES DE LOS SECTORES PÚBLICO Y PRIVADO DE PUERTO RICO, COLOMBIA Y COSTA RICA
}

\author{
Hernán A. Vera Rodríguez ${ }^{1}$ \\ Jennifer Isabel Arroyo Chacón ${ }^{2}$ \\ Ana Fernanda Uribe Rodríguez ${ }^{3}$ \\ Jennifer Castellanos Barreto ${ }^{4}$
}

Recibido: 30/05/2018

Aprobado: $31 / 08 / 2018$

RESUMEN

Se presenta un estudio con metodología mixta predominantemente cuantitativa, el cual tuvo como propósito identificar la forma en que actuarían los gerentes en situaciones hipotéticas relacionadas con el soborno, coerción, engaño, robo, discriminación y transparencia. La muestra estuvo compuesta por 281 gerentes de los sectores público y privado de Puerto Rico, Colombia y Costa Rica. Para recolectar la información, se usó la versión en español del Cuestionario Sobre Situaciones Éticas de Fritzsche y Oz (2007). Se realizaron análisis estadísticos descriptivos, pruebas t para muestras independientes, Anova de una dirección y análisis de contenido en el que se agruparon las respuestas cualitativas por categorías. Se identificó que, en la mayoría de los casos, los gerentes encuestados tomarían una decisión ética ante un escenario éticamente ambiguo. Así mismo, se encontraron diferencias estadísticamente significativas entre los gerentes de los sectores público y privado de algunos de los países estudiados en determinadas situaciones éticas. En términos generales, se observaron tendencias éticas ligeramente más fuertes entre los gerentes del sector público que entre los del sector privado. Por otro lado, no se encontraron diferencias estadísticamente significativas en las tendencias éticas de los gerentes que formaron parte de la investigación, por razón de su país donde trabajaban: Puerto Rico, Costa Rica o Colombia. Finalmente, los resultados cualitativos revelaron que el razonamiento axiológico, fue el tipo de razonamiento más utilizado por los gerentes de la muestra, al enfrentar un dilema o situación éticamente ambigua.

PALABRAS CLAVE: ORGANIZACIÓN, TOMA DE DECISIONES, JUICIO DE VALOR, ADMINISTRACIÓN DE EMPRESAS,ADMINISTRACIÓN PÚBLICA.

CLASIFICACIÓN JEL: M500, M540, P520

1 Pontificia Universidad Católica de Puerto Rico, Colegio de Estudios Graduados en Ciencias de la Conducta y Asuntos de la Comunidad; Código Postal 00717; Ponce, Puerto Rico; hvera@pucpr.edu

2 Universidad Estatal a Distancia (UNED); Código Postal, 1174 - 1200; San José, Costa Rica; info@profesorajenniferarroyo.com ; prof.jenniferarroyo@yahoo.com

3 Universidad Pontificia Bolivariana, Seccional Bucaramanga; Código Postal: 50031; Santander, Colombia; anafernanda.uribe@ upb.edu.co

4 Pontificia Universidad Católica de Puerto Rico, Colegio de Estudios Graduados en Ciencias de la Conducta y Asuntos de la Comunidad. Departamento de Psicología Industrial - Organizacional. Apartado Postal: 00717. 2250; Ponce, Puerto Rico; jcastellanosbarreto@pucpr.edu 


\begin{abstract}
A mixed methodology study, with a quantitative emphasis is presented. Its main purpose was to identify the way in which managers would act in hypothetical situations related to bribery, coercion, cheating, theft, discrimination and transparency. The sample consisted of 281 managers from public and private organizations in Puerto Rico, Colombia and Costa Rica. Data and information gathering was performed using the Spanish version of the Ethical Situations Questionnaire developed by Fritzsche and $\mathrm{Oz}$ (2007). Descriptive statistical analyzes, t-tests for independent samples and one way Anova's were used for the analysis of quantitative data. On the other hand, content analysis in which the qualitative responses were grouped by categories was used to analyze qualitative information. Results show, that in most cases, sampled managers would take an ethical decision in an ethically ambiguous scenario. Results showed significant statistical differences between the ethical tendencies of public and private sector managers in some of the ethical situations. In general terms, slightly stronger ethical trends were observed among public sector managers. On the contrary, the study did not find statistically significant differences due to the country in which they worked. Finally, the axiological reasoning was the type of reasoning mostly used by surveyed managers, when faced with an ethically ambiguous dilemma or situation.
\end{abstract}

KEYWORDS: LABOR, CONDITIONS OF EMPLOYMENT.

JEL CLASIFICATION: M500, M540, P520

\title{
I. INTRODUCCIÓN
}

Desde sus orígenes, la administración pública y la administración de empresas o de negocios han estado estrechamente vinculadas. De este modo, en el Estado moderno, la primera se encarga de fijar las "reglas de juego" para el establecimiento de empresas en las sociedades democráticas, así como de facilitar su origen y florecimiento, pues se considera que estas ayudan al crecimiento económico de los países y propician el bienestar de grandes sectores de la sociedad (Furlong, 2003; Griorescu, 2006). Así mismo, también regula a las empresas mediante mecanismos tales como: legislación antimonopolios, legislación de salario mínimo, de jornada laboral y de prestaciones, así como otro tipo de protecciones legales del trabajo y los trabajadores (Furlong, 2003). También, ha reglamentado el comercio entre países y ha establecido la "cuota social" que cada negocio aportará a la sociedad mediante la legislación impositiva. Asimismo, ha establecido mecanismos para proteger las invenciones y la propiedad intelectual mediante procesos de obtención de patentes y derechos de autor, entre otros (Boex, 2013; Pandey, 2006).

Pese a los esfuerzos que se hacen a diario -en sus propios ámbitos de acción-, las organizaciones de tipo privado o público siguen prevaleciendo el soborno, los actos de corrupción impunes y la falta de respuesta a las necesidades de los ciudadanos. De hecho, de acuerdo con el Índice de Percepción de la Corrupción (IPC), en el 2016, el 69 \% de los 176 países incluidos en este indicador obtuvieron una puntuación inferior a 50 puntos, en una escala que va de cero (percepción de altos niveles de corrupción) a 100 (percepción de ínfimos niveles de corrupción), lo cual deja ver la percepción generalizada de la corrupción en el sector público a nivel mundial.

Durante ese año, Colombia tuvo un índice de 37 puntos y la posición número 90 entre 176 países que formaron parte del estudio. Cabe notar que mejoró su posición jerárquica con relación a otros países, pues ocupaba el puesto número 94 en el año 2014, aunque su índice de percepción de la corrupción se mantuvo en 37 puntos, lo cual implica una percepción de corrupción de moderada a alta. En el caso de Costa Rica, el índice ha mejorado progresivamente, ya que logró el puesto número 41 en el 2016, habiendo estado en el puesto 48 para el 2014 (Transparency International 
Secretariat, 2017). Asimismo, su Índice de Percepción de Corrupción mejoró al obtener 54 puntos en el 2014, y 58 puntos en el 2016. A pesar de esto, los índices implican una percepción ciudadana de unos niveles moderados de corrupción. Por otra parte, Puerto Rico no presenta IPC para los años 2016 y 2015 por razones que desconocen los investigadores. Sin embargo, se encuentra en el reporte del 2014, en el cual obtuvo el puesto número 31 entre 174 países. Logró, asimismo, un IPC de 63 puntos, lo cual implica una percepción moderada de corrupción (Transparency International Secretariat, 2014).

A tono con los esfuerzos por promover la ética y combatir la corrupción, el Centro Latinoamericano de Administración para el Desarrollo (CLAD,2006) ha establecido varios documentos y cartas doctrinales; entre ellos se destacan el Código Iberoamericano de Buen Gobierno y la Carta Iberoamericana de Calidad en la Gestión Pública (CLAD, 2008).

En primer lugar, en el Código Iberoamericano de Buen Gobierno (CLAD, 2006), los países iberoamericanos firmantes se comprometieron a que los miembros del Poder Ejecutivo de sus respectivos Gobiernos evitarían la corrupción, y:

Se abstendrán de toda actividad privada que pueda constituir un conflicto de interés con su cargo público...no aceptarán ningún trato de favor o situación que implique un privilegio o ventaja injustificada...rechazarán cualquier regalo, favor o servicio, personal o familiar, en condiciones ventajosas que más allá de los usos habituales, sociales y de cortesía, puedan condicionar el desempeño de sus funciones...no influirán en la agilización o resolución de trámite o procedimiento administrativo sin justa causa..., y evitarán todo uso indebido de información que conozcan por razón de su cargo. (CLAD, 2006, pp.5-7)

Igualmente, el Código plantea el rol que pueden desempeñar los funcionarios del Poder Ejecutivo en partidos políticos u organizaciones similares, el cual "en ningún caso menoscabará o comprometerá el ejercicio eficaz e imparcial de sus funciones” (CLAD, 2006, p. 7).

En segundo lugar, en la Carta Iberoamericana de Calidad en la Gestión Pública (CLAD, 2008) se detallan, entre otros, los principios de "transparencia y participación ciudadana" y el de "ética pública" como algunos de los principios inspiradores de una gestión pública de calidad. Sobre el primer binomio de estos, la Carta apunta que: "las Administraciones Públicas tienen que ser transparentes en su gestión, abiertas al escrutinio público, tolerantes a la crítica y permeables a las propuestas de mejora y de cambio provenientes de la ciudadanía" (CLAD, 2008, p.9). Por otra parte, respecto al principio de la ética pública, este documento doctrinal puntualiza que:

Una gestión pública de calidad implica la observancia de valores éticos que la orientan, tales como la vocación de servicio público, la probidad, la honradez, la buena fe, la confianza mutua, la solidaridad y la corresponsabilidad social, la transparencia, la dedicación al trabajo, el respeto a las personas, la escrupulosidad en el manejo de los recursos públicos y la preeminencia del Interés público sobre el privado. (CLAD, 2008, p.8)

A tenor con lo planteado en estos documentos, es sabido que, en los países iberoamericanos, en nuestro caso, Puerto Rico, Colombia y Costa Rica, tanto las organizaciones del sector público como las del sector privado han estado muy activas -en sus ámbitos particulares de acción- en el desarrollo de normas, leyes, organismos y procesos con el propósito de promover la ética y combatir la corrupción. De igual forma, es relevante señalar que la promoción de la ética gubernamental y el combate contra la corrupción es una agenda que requiere continuarse. De la premisa anterior surge la motivación y justificación para este estudio. A continuación, se presenta la revisión bibliográfica de este. 
Dentro de las primeras investigaciones que se hallan en relación al tema, se encuentra un estudio llevado a cabo por Van Der Wal, Hubers, Den Heuvel y Kolthoff (2006), en Holanda. Estos autores realizaron un análisis de la literatura periódica sobre la ética administrativa en el sector público y la ética empresarial, así como de los códigos de ética de organizaciones públicas y privadas de su país. Resulta interesante su planteamiento de que "la ética administrativa pública y la ética empresarial parecen ser mundos separados: sin embargo, los intentos por llevar a cabo estudios interdisciplinarios y comparativos han sido escasos" (p. 321). Estos autores determinaron que, en la bibliografía periódica de la administración pública, los valores centrales de la gestión gubernamental son la honestidad, el humanismo, la justicia social, la imparcialidad, la transparencia, la integridad, la obediencia, la confiabilidad, la responsabilidad, el profesionalismo, la rendición de cuentas, la eficiencia y el coraje o la determinación.

Por otra parte, entre los valores centrales de la administración de empresas, los autores hallaron los siguientes: la honestidad, orientación al cliente, profesionalismo, confiabilidad, trabajo en equipo, empresarismo, responsabilidad social, innovación, autoeficacia, efectividad, maximización de rendimientos y sostenibilidad. Al comparar estos valores centrales que están presentes en los códigos de conducta de cerca de 200 compañías privadas multinacionales, los autores hallaron muchas similitudes y algunas diferencias con los valores anteriormente presentados y los cuales surgen de la bibliografía periódica de administración de empresas. Interesantemente, puntualizan la ausencia del valor de integridad en los códigos de ética o conducta de las compañías privadas evaluadas y destacan la posibilidad de que algunos conceptos muy cercanos se designen de forma distinta en ambos sectores, lo cual puede dar lugar a las aparentes diferencias.

En otro estudio, Chun (2009) comparó los valores éticos y las tendencias de protección del ambiente de empleados de organizaciones públicas y privadas de siete compañías relacionadas con la minería de carbón para producción de energía en una de las regiones mineras más grandes de China, y la cual genera una gran cantidad de contaminación.

Entre los resultados de la investigación, se destacan los siguientes. En primer lugar, se determinó que hubo diferencias estadísticamente significativas en los resultados relativos a la ciudadanía corporativa, entendida como el rol o la responsabilidad de una organización para con la sociedad, los valores de los empleados y las actitudes sobre el ambiente por razón del tipo de organización, pública o privada, a la cual pertenecían los empleados. Paradójicamente, se encontró que los empleados de corporaciones públicas tenían niveles más bajos de ciudadanía corporativa (económica, legal, ética y discrecional) que sus contrapartes del sector privado. También, la investigadora halló resultados similares respecto a las actitudes hacia la protección del ambiente: los empleados públicos encuestados tuvieron unas actitudes más pobres hacia la protección del ambiente que los participantes del sector privado. De forma similar, los empleados públicos tuvieron puntuaciones más bajas en la escala de valores que sus homólogos del sector privado. Finalmente, la autora refiere que el Gobierno chino no ha desempeñado adecuadamente su rol de promover actitudes positivas y productivas entre sus empleados del sector minero dedicado a la producción de energía.

Entre otros estudios sobre la ética en los que se utiliza una metodología similar a la utilizada en el presente estudio, se encuentra el de Fritzsche, Huo, Sugai, Dun-Hou, Seok \& Becker (1995). Estos autores examinaron los indicadores de comportamientos éticos mediante el estudio de participantes de cuatro países: Estados Unidos, Japón, Corea del Sur y Taiwán, y recopilaron los datos de su estudio utilizando un cuestionario compuesto por una serie de cinco situaciones hipotéticas ("vignnettes") en apariencia ambiguos en términos éticos. Los participantes contestaron a cada situación indicando qué acción hubieran tomado y la razón que justificaba su decisión. Los temas representados en el instrumento, a modo de dilemas de naturaleza ética, fueron: la coerción y el control, el conflicto de intereses, el ambiente físico, el paternalismo y la integridad personal. 
En el estudio (Fritzsche et al., 1995) participaron gerentes de los cuatro países $(\mathrm{N}=$ 316), en su mayoría varones con educación universitaria, que trabajaban en la industria de la manufactura. Asimismo, fungían como gerentes de nivel intermedio. En la investigación se analizaron las respuestas de los participantes referentes a la acción que llevarían a cabo según la situación presentada en el dilema. Además, se construyeron categorías para agrupar las justificaciones planteadas por los participantes para su acción o determinación.

Los resultados indicaron que no hubo diferencias estadísticamente significativas por país en cuanto a la acción referente a la coerción y el control (soborno). Sin embargo, el análisis cualitativo de las explicaciones de los encuestados respecto a estos dos dilemas mostró que, para una porción de los encuestados, la dimensión económica de la decisión fue el elemento más importante a la hora de tomar la misma, considerando ellos el soborno como una "inversión". Otro resultado importante fue que la mayoría de los participantes no demostró la posibilidad de incurrir en comportamientos dañinos al ambiente. La ilegalidad de esta acción fue la justificación principal argumentada por los participantes para no incurrir en ella. Por otro lado, en el dilema sobre paternalismo, la mayoría de los participantes respondió tomando en consideración el bienestar y la seguridad de otros (Fritzsche et al., 1995).

Los autores concluyeron que la justificación para tomar decisiones morales ante una situación ambigua variará de acuerdo con la cultura y con los principios éticos envueltos en el dilema. Concluyeron, además, que la toma de decisiones ética de los gerentes japoneses parece ser más parecida a la de los norteamericanos que a la de las otras dos nacionalidades. Además, tanto los gerentes japoneses como los estadounidenses presentaron una mayor preocupación por los aspectos legales y los riesgos económicos de sus acciones que el resto de los participantes (Fritzsche et al., 1995).

Por su parte, Lim (2003) exploró los efectos de moderación que parecen tener los intereses personales $y$ organizacionales sobre el juicio ético de una muestra compuesta por 397 participantes, la cual incluía tres grupos: chinos residentes en China, chinos residentes en Singapur y personas naturales de Singapur residentes en dicho país. El investigador halló que los intereses organizacionales tuvieron efectos altamente significativos en el juicio ético de los chinos residentes en China, de los chinos residentes en Singapur, pero no en singapurenses que residían en Singapur. Por otra parte, los intereses individuales no demostraron tener un efecto significativo en el juicio ético de los tres grupos estudiados en comparación con el grupo control utilizado en el estudio.

Asimismo, los resultados revelaron que, cuando se excluían los intereses propios y organizacionales del análisis, los chinos residentes en China denotaban apego a los estándares éticos más altos que los otros dos grupos, mientras que cuando se consideraban únicamente los intereses personales en el mismo, los chinos residentes en Singapur denotaban los estándares éticos más bajos. En contraste, cuando se consideraban los intereses organizacionales, los chinos residentes en China demostraban tener unos estándares éticos más bajos que los singapurenses residentes en Singapur.

Según Lim (2003), tales resultados apuntan a que los chinos demuestran mayor flexibilidad o maleabilidad en sus estándares éticos que los singapurenses. Asimismo, sugieren que los individuos, en ambientes socio-culturales distintos, exhibirán patrones de juicio ético en reacción a los intereses propios y organizacionales.

En esta línea de investigaciones internacionales sobre el razonamiento ético, se encuentra un estudio realizado por Alavi, Yasin y Zimmerer (2004). Estos examinaron si los valores de los ejecutivos iraníes contemporáneos eran más consistentes con una orientación filosófica deontológica (normativa o guiada por principios morales o éticos universales) o con una orientación filosófica pragmática o utilitaria. A los entrevistados se les administró un cuestionario basado en los trabajos de Kohlberg sobre el razonamiento moral. 
Los resultados del estudio apuntan a que la orientación de los valores de los ejecutivos iraníes es una principalmente deontológica, basada en valores religiosos, aunque parecen estar en un período de transición hacia valores morales más universales y globales. Los autores apuntan que los ejecutivos iraníes parecen estar experimentando altos niveles de conflicto entre los valores éticos y morales musulmanes tradicionales y los valores requeridos para lograr competitividad en un mercado global.

Por otra parte, existen varios estudios en los que se aplica la corriente metodológica establecida por Fritzsche et al. (1995) y continuada por los estudios antes reseñados para comparar las disposiciones éticas entre gerentes y ejecutivos de distintos países de las disposiciones éticas de gerentes de los sectores público y privado. El primero es el de Wheeler y Brady (1998). La muestra de este estudio estuvo compuesta por empleados (no gerenciales) de una empresa del sur de los Estados Unidos de América y por empleados (no gerenciales) de una compañía financiera. A los participantes, se les administró un cuestionario compuesto de ocho situaciones éticas hipotéticas (o vignettes), a las cuales debían responder mediante respuestas de selección múltiple. De cuatro posibilidades de respuesta, dos eran de naturaleza deontológica y dos de naturaleza utilitaria.

Las conclusiones del estudio revelaron que los participantes se inclinaron más por las soluciones deontológicas a los dilemas éticos presentados. Asimismo, no se encontraron diferencias estadísticamente significativas entre los empleados de los sectores público y privado. Sin embargo, se halló que los empleados del sector privado tenían una ligera inclinación a favorecer más los razonamientos éticos utilitarios u ontológicos que sus homólogos en el sector público.

Otro estudio es el llevado a cabo por Roozen, Pelsmacker y Bostyn (2001), el cual tuvo una muestra de 427 graduados de economía de una universidad belga. Estos completaron un cuestionario enviado por correo en el que evaluaron una serie de frases o situaciones relacionadas con la ética en una escala de cero a diez. Los resultados evidenciaron que los participantes que trabajaban en las áreas de servicios públicos y la educación, tendieron a inclinarse más hacia los aspectos éticos del proceso decisorio. Por otro lado, aquellos que laboraban en la banca y la industria de los seguros tuvieron una inclinación menor hacia los aspectos éticos del proceso decisorio. De forma similar, se halló que quienes trabajaban en industrias en las que, según su criterio, la meta de la maximización de la tasa de ganancias era muy importante, tuvieron puntuaciones más bajas en cuanto a los aspectos éticos del proceso decisorio que aquellos que laboraban en las cuales la tasa de ganancia era menos importante.

En esa misma línea se encuentra una investigación llevada a cabo por Khuntia y Suar (2004), en la cual se administró una escala de 22 reactivos a 340 gerentes de nivel intermedio y a sus superiores. Los resultados señalan que los superiores tendieron a otorgarse puntuaciones superiores en la escala de liderazgo ético, en comparación a la otorgada por los subordinados. De igual forma, indicaron que los participantes que laboraban en el sector público evaluaron el liderazgo de sus superiores como uno más ético que los participantes que laboraban en el sector privado.

Los estudios anteriores son el marco referencial de la presente investigación. Como variables independientes se consideraron el tipo de sector laboral (público y privado) y los tres países de donde eran los participantes (Puerto Rico, Colombia y Costa Rica). Como variable dependiente se tuvo en cuenta las respuestas de los participantes al Cuestionario de Situaciones Éticas de Fritzsche y Oz (2007), según modificado por Vera (2016) y Ortiz (2016). Por lo tanto, las hipótesis de trabajo del estudio fueron las siguientes:

- H1 - Existen diferencias estadísticamente significativas en el promedio de las respuestas de los gerentes en las escalas del Cuestionario de Situaciones Éticas de Fritzsche \& Oz (2007) por razón del sector laboral (público o privado).

- H2 - Existen diferencias estadísticamente significativas en la puntuación promedio del Cuestionario de Situaciones Éticas de Fritzshe \& Oz (2007) por razón del país en el cual se desempeñan los gerentes (Puerto Rico, Colombia o Costa Rica). 


\section{METODOLOGÍA}

\section{Participantes}

La muestra estuvo compuesta por 281 gerentes (altos directivos, gerentes, sub-subgerentes y líderes de equipo) de los sectores públicos y privados de Puerto Rico, Colombia y Costa Rica. La submuestra de Puerto Rico estuvo constituida por 106 gerentes, los cuales provenían de múltiples municipios de Puerto Rico; la submuestra de Colombia abarcaba 79 gerentes, seleccionados principalmente de la ciudad de Bucaramanga del departamento de Santander. Por otra parte, la submuestra de Costa Rica consistió en 96 gerentes y se seleccionó principalmente en la ciudad de San José. Con relación al sector laboral (público o privado), en el caso de Puerto Rico, 50 de los participantes provenían del sector público y 56 del sector privado; en Colombia, 32 de los gerentes laboraban en el sector público y 47 en el sector privado; mientras, en Costa Rica, 59 eran del sector público y 37 del sector privado.

Los participantes fueron seleccionados por conveniencia o por referido, de una de las siguientes formas:

a. se les envió una invitación al correo electrónico porque fueron referidos por facultativos, estudiantes o egresados de las universidades en las cuales laboran los investigadores;

b. se les envió la invitación por correo electrónico a participantes utilizando información de contacto provista por colegios y asociaciones profesionales; $y$

c. se hizo contacto personal con ellos a través de referidos de las fuentes antes mencionadas.

Este tipo de muestreo es muy útil cuando no existe un marco muestral, un listado o base de datos con los nombres e información de contacto de todos los sujetos de una población, y cuando resulta muy difícil el seleccionar los mismos de forma aleatoria.

En cuanto a las características sociodemográficas de la muestra, en el caso de Puerto Rico, esta estuvo compuesta por gerentes con edades que fluctuaban entre los 22 y los 64 años con una edad promedio de 43 años y una desviación estándar de 10,15 años. En términos educativos, el $36,8 \%$ contaba con un bachillerato (pregrado), el 44,3\% con una maestría (grado), el 7,5\% con un doctorado. Igualmente, el 5.7\% tenía un diploma de escuela superior (secundaria) y el 5,7\% tenía otro tipo de grado (grado asociado, grado técnico).

Por su parte, la submuestra de Colombia también fue integrada por personal gerencial que se hallaba en un rango de edad entre 23 a 75 años, con un promedio de 41,9 años y una desviación estándar de 10,64 años. En términos educativos, el 24,1\% contaba con un pregrado (bachillerato), el $40,5 \%$ con especialización, el 31,6\% con maestría, el 2,5\% con doctorado y el 1,3\% con formación tecnológica.

Finalmente, la submuestra de Costa Rica también estuvo conformada por gerentes, quienes tenían una edad promedio de 47,07 años con una desviación estándar de 9,74 años. La edad mínima fue de 23 años y la máxima de 68 años. En términos de su preparación académica, el 3,1\% poseía un bachillerato, el 34,4\% una licenciatura, el 42,7\% una maestría y el 4,2\% un doctorado. Quince participantes $(15,6 \%)$ no contestaron esta pregunta.

\section{Instrumento}

Se utilizó la versión en español del Cuestionario Sobre Situaciones Éticas de Fritzsche y Oz (2007), el cual se adaptó de acuerdo a las necesidades de este estudio (ver anexo A). En específico, se anadió un nuevo dilema, el de la transparencia, por ser un tema de gran importancia y actualidad, 
tanto en la administración pública, como en la gerencia, y por no estar incluído en el cuestionario original de los autores. Asimismo, se añadió una pregunta de seguimiento relacionada a este dilema, para hacerlo cónsono con las preguntas del cuestionario original. Durante el desarrollo de una investigación anterior, Vera y Pietri (2009) obtuvieron el permiso del autor para traducir y validar la escala al español. Se aseguró la equivalencia entre el instrumento en español y el original en inglés mediante el método de traducción en reversa (backward translation). Se obtuvo un valor de .90 para la concordancia entre las dos versiones del instrumento.

En dicho trabajo, se determinó el coeficiente de confiabilidad del instrumento mediante el método de alfa de Cronbach, y se obtuvo como resultado un coeficiente de .572 . El instrumento constaba de cinco situaciones hipotéticas, pero plausibles (y un tanto ambiguas en términos éticos), en las que se presentaban dilemas éticos relacionados con las áreas de: soborno, coerción, engaño, robo y discriminación ilegal en el trabajo. Para actualizar esta escala y adaptarla a su uso en la administración pública, se añadió un dilema relacionado con la transparencia desarrollada por Vera (2016) y Ortiz (2016). Los participantes indicaban primero la acción que tomarían en cada situación, mediante respuesta en una escala de diez puntos que iba desde cero ("definitivamente no lo haría"), hasta diez ("definitivamente lo haría"). Luego, en un espacio provisto para ello, explicaban las razones por las cuales daban sus respuestas a las seis situaciones presentadas en el cuestionario. Se calculó un índice agregado para cada una de las seis situaciones hipotéticas tanto para los participantes que laboraban en el sector público, como para los que laboraban en el sector privado. Vale la pena notar que cuando la direccionalidad de la escala de la situación número cinco (discriminación ilegal) era distinta a las de las demás escalas, se llevó a cabo una inversión de los resultados de cada participante (reverse scoring) para homologarlos a los de las otras cinco escalas. Asimismo, se calculó un índice agregado para las seis situaciones hipotéticas para cada país.

Luego, se llevaron a cabo análisis estadísticos descriptivos, así como pruebas t para muestras independientes para determinar si había diferencias estadísticamente significativas en las puntuaciones promedio de los participantes del sector público y los del sector privado. Asimismo, se llevaron a cabo análisis de varianza (ANOVA de una dirección) para determinar si había diferencias estadísticamente significativas en los promedios de los participantes en cada dilema por razón del país en el cual se desempeñaban (Puerto Rico, Colombia y Costa Rica). Más adelante, se realizó un análisis cualitativo de las explicaciones que dieron los participantes para sus respuestas a cada uno de los dilemas mediante la técnica de análisis de contenido. Así pues, se agruparon por categorías y se determinó si sus explicaciones al dilema ético eran de naturaleza deontológica, axiológica, utilitarista, relativista o de otro tipo.

Aunque los autores evaluaron múltiples posibilidades de clasificación de las respuestas de los participantes, optaron por la siguiente. En primer lugar, se clasificaron como deontológicas, aquellas explicaciones de los participantes en las cuales se actuaba (o no) de una manera ética porque la situación planteada se relacionaba con algún código, reglamento, ley, estatuto, etc. Por otro lado, las explicaciones se calificaron como axiológicas cuando se relacionaban con razones de conciencia, valores, creencias, así como con las normas o mores culturales. Finalmente, si en el análisis de contenido de las respuestas predominaba la búsqueda de beneficios para sí mismo, para su núcleo familiar, para la organización laboral o sus miembros, se calificaba la respuesta como una utilitaria, esta designación también aplicaba a aquellas explicaciones en las cuales predominaba la intención de evitar un castigo o pena. En el caso de aquellas respuestas cuyo análisis denotara un relativismo o ambivalencia ética, se denominaban como relativistas. Por último, aquellas explicaciones que no se adaptaban a las categorías anteriores se agruparon bajo la categoría de "otra".

Además de este instrumento, se incluyó una planilla de datos sociodemográficos en la cual los participantes indicaban el tipo de industria en la que laboraban (sector público o sector privado), su edad, el grado académico más alto obtenido. Estas últimas preguntas se frasearon de este modo, para evitar advertir a los participantes que la ética era el foco del estudio. Las alternativas de las 
preguntas incluidas en la planilla sociodemográfica se atemperaron a la realidad de cada uno de los países en los que se llevó el estudio.

\section{Procedimiento}

Se prepararon versiones del cuestionario para cada país en Google Documents, con el propósito de enviarlas a los participantes por correo electrónico. Esto se llevó a cabo de forma que se garantizara la confidencialidad de los participantes, al no guardarse la dirección electrónica (IP Address), ni ningún otro identificador de los participantes. Las respuestas se recibían directamente al Google Drive de los investigadores de cada país, y se recibía una notificación por correo electrónico de la llegada de estos. Estas respuestas eran luego descargadas a un archivo de Microsoft Excel. Asimismo, se administraron también los instrumentos físicamente (en papel) por los investigadores y en algunos casos, por asistentes de investigación, debidamente adiestrados por los investigadores.

Luego, los resultados de los cuestionarios que llegaron por Internet fueron descargados a archivos de MS Excel y exportados al Programa IBM SPSS, Versión 23. Asimismo, los cuestionarios que se recibieron de forma física fueron tabulados e incorporados en estas bases de datos. Se llevaron a cabo los análisis estadísticos, tanto descriptivos como inferenciales (pruebas t para muestras independientes, tabulaciones cruzadas) para cada país. Además, se llevó a cabo un análisis de varianza unidireccional para determinar si había diferencias significativas en los promedios obtenidos por los participantes en cada dilema por razón de su país de procedencia. Más adelante, se analizó el contenido de las explicaciones que dieron los participantes de cada país para determinar si sus juicios éticos en cada situación planteada eran de naturaleza deontológica, axiológica, utilitarista, relativista o de otro tipo.

\section{RESULTADOS}

\section{Resultados descriptivos sobre tendencias éticas}

Al tabular los resultados para cada dilema, se consideraron aquellas puntuaciones entre cero al cuatro como equivalentes a que el participante no incurriría en dicho dilema, mientras que aquellas de cinco al diez se tomaron como indicativas de que los participantes incurrirían en la conducta presentada en el dilema. Asimismo, se analizaron las puntuaciones en los extremos de cada escala (definitivamente no lo haría (cero) o definitivamente lo haría (10), para ahondar más en el análisis de las tendencias éticas de los participantes.

Los resultados del primer dilema correspondiente a soborno. En este caso, se identificó que el 96,2\% de los participantes puertorriqueños, el 96,2\% de los colombianos y el 98,1\% de los costarricenses no incurriría en dicha conducta. En contraste, el 3,8\% de los participantes de Puerto Rico, el 3,8\% de los de Colombia y el 3,1\% de los de Costa Rica manifestaron que incurrirían en dicha práctica de acuerdo con los hechos planteados. Para indagar más en los resultados, se evaluó el porciento de participantes que marcaron cero (definitivamente no incurría en dicha práctica) o diez (definitivamente incurriría en dicha práctica) en la Escala Likert provista para este dilema. Se encontró que el 84,9\% de los participantes puertorriqueños, el 84,8\% de colombianos y el 91,6\% de los costarricenses manifestaron que definitivamente no aceptarían un soborno. Por otro lado, ningún participante de Puerto Rico (0\%), el 2,5\% de los de Colombia y el 1,1\% de los de Costa Rica apuntaron que definitivamente lo harían.

Al examinar los resultados del segundo dilema, la coerción, se halló que el 98,1\% de los gerentes puertorriqueños, el 96,2\% de los colombianos y el 99,0\% de los costarricenses declararon que no coaccionarían a un subordinado para que llevara a cabo una acción antiética, tal como presentada en el segundo dilema del estudio. No obstante, los resultados respecto a los participantes 
puertorriqueños refieren que en contraste, el 0,9\% incurriría en esta práctica según la situación administrativa presentada, $y$ un participante $(0,9 \%)$ no ofreció su contestación a este dilema. Asimismo, se encontró que el 3,8\% de los gerentes colombianos y 1,0\% de los costarricenses manifestaron que coaccionarían a un subordinado para que realizara acciones antiéticas para su beneficio. De forma similar, al revisar los extremos de la Escala Likert, se pudo notar que el 90,6\% de los encuestados puertorriqueños apuntaron que definitivamente no coaccionarían a un subordinado para que llevara a cabo una acción antiética, mientras que ninguno apuntó que definitivamente lo haría. Por su parte, el 92,4\% de los gerentes colombianos y el 99,0\% de los costarricenses indicaron que definitivamente no coaccionarían a un subordinado para que este realizara acciones antiéticas en su nombre. En contraste, el 2,5\% de los participantes de Colombia y ninguno de los participantes de Costa Rica afirmaron que definitivamente coaccionarían a un subordinado para que llevara a cabo la acción presentada en el mencionado dilema del cuestionario.

Respecto al tercer dilema, la decepción o engaño, los resultados presentaron que el $63,2 \%$ de los participantes puertorriqueños, el 50,6\% de los colombianos y el 68\% de los costarricenses indicaron que no falsearía información sobre un producto o servicio para salvar la organización para la cual trabajan. En contraste, en el caso de Puerto Rico, el 34,9\% lo haría. Dos participantes (1,9\%) no ofrecieron respuesta a este dilema. De forma similar, el 49,4\% de los participantes colombianos y el 31,3\% de los costarricenses también apuntaron que falsearían información sobre un producto o servicio para salvar su organización laboral. Al examinar los resultados en los extremos de la Escala Likert, se halló que el 47,2\% de los participantes puertorriqueños, el 31,6\% de colombianos y el $49,0 \%$ de los costarricenses indicaron que definitivamente no incurrirían la práctica del engaño, lo cual quedó patentizado al seleccionar el cero en la escala provista, mientras que el 9,4\% de los gerentes puertorriqueños, el 11,4\% de los colombianos y el 5,2\% de los costarricenses manifestaron que definitivamente lo harían.

En el cuarto dilema, se presentaba el robo como una situación organizacional ambigua. En este, el 84,9\% de los gerentes puertorriqueños, el 86,1\% de los colombianos y el 80,2\% de los costarricenses que formaron parte de la muestra, manifestaron que no incurrirían en dicha práctica. En contraste, el 13,2\% de los participantes de Puerto Rico, el 13,9\% de los de Colombia, y 19,8\% de los de Costa Rica contestaron de forma afirmativa. El 1,9\% de los participantes de Puerto Rico no se expresó respecto a este dilema. Al examinar los resultados en los extremos de la escala Likert, se pudo notar que el 70,2\% de los participantes puertorriqueños, el 70,9\% de los colombianos y el 68,85 de los costarricenses indicaron que definitivamente no incurriría en esta práctica, pues otorgaron cero en la Escala Likert Relativa a este dilema. En contraposición, el 3,8\% de los gerentes de Puerto Rico, el 3,8\% de los de Colombia y el 2,1\% de los de Costa Rica apuntaron que definitivamente lo harían, lo cual se manifestó por haber marcado el número 10 (máxima puntuación) como su contestación en la Escala Likert provista en el cuestionario.

Con relación al quinto dilema sobre la discriminación, el 53,8\% de los participantes puertorriqueños, el 51,9\% de los colombianos y el 58,3\% de los costarricenses manifestaron no inclinarse a discriminar por razón de origen nacional en el empleo. Por el contrario, el 46,2\% de los gerentes de Puerto Rico, el 48,1\% de los de Colombia, y el 41,7\% de los de Costa Rica ofrecieron respuestas que denotaban su inclinación a esta práctica. Por otra parte, al examinar los extremos de la escala, se encontró que el $26,4 \%$ de los participantes puertorriqueños, el 31,6\% de los colombianos y el $29,2 \%$ de los costarricenses manifestaron que definitivamente no discriminarían en la contratación de un empleado por razones de origen nacional. En contraposición, el 2,8\% de los encuestados puertorriqueños, el 16,5\% de los colombianos y el 2,1\% de los costarricenses manifestaron que definitivamente discriminarían por razón de origen nacional en un proceso de contratación como el planteado en el quinto dilema del cuestionario.

Por último, en los análisis del sexto dilema acerca de la transparencia, se identificó que el $62,3 \%$ de los gerentes puertorriqueños encuestados, así como el 63,3\% de los colombianos y el 
$58,9 \%$ de los costarricenses no negarían a la prensa y a la ciudadanía información relacionada a la destrucción del patrimonio cultural del país. En contraste, el 35,8\% de los participantes de Puerto Rico, el 36,7\% de los colombianos, y el 41,1\% de los de Costa Rica, negaría la existencia de dicha información. Dos participantes (1,9\%) no ofrecieron sus respuestas a este dilema. Al analizar las respuestas en los extremos de la escala Likert de esta pregunta, se halló que el 48,1\% de los participantes puertorriqueños, el 49,4\% de los colombianos y el 41,1\% de los costarricenses definitivamente no negarían la existencia de la información solicitada, es decir, serían transparentes en su gestión. Por otra parte, se halló que el 8,5\% de los gerentes puertorriqueños, el 7,6\% de los colombianos y el 12,8\% de los costarricenses señalaron que definitivamente negarían la existencia de documentos comprometedores a la prensa y a la ciudadanía, es decir, que sus actuaciones no serían transparentes.

\section{Análisis inferenciales sobre tendencias éticas: sectores público y privado}

Otra serie de análisis estadísticos llevados a cabo se encaminó a someter a prueba la hipótesis principal del presente estudio, la cual planteaba que habría diferencias estadísticamente significativas en las disposiciones éticas de los gerentes del sector público y los del sector privado que formaron parte de la muestra del estudio.

Respecto al primer dilema, el soborno, en los análisis de prueba t para muestras independientes no revelaron diferencias estadísticamente significativas entre las puntuaciones de los participantes de los sectores público y privado de Puerto Rico, Colombia ni Costa Rica. Un examen de los promedios obtenidos por los participantes de ambos sectores de la economía reveló que los participantes del sector privado tuvieron una mayor inclinación por respuestas en las que se aceptaba la práctica del soborno en los tres países de los cuales se obtuvo la muestra del estudio (ver cuadro 1).

Por otro lado, en relación con el segundo dilema ético sobre la coerción, en los resultados de la prueba $t$, no se encontraron diferencias estadísticamente significativas en las puntuaciones de los gerentes de los sectores público y privado de Puerto Rico, Colombia y Costa Rica que formaron parte de la investigación. Vale la pena notar que en este dilema se hallaron puntuaciones promedio más altas para los gerentes del sector privado en la submuestra de Puerto Rico, pero no así en las de Colombia y Costa Rica, en las cuales los gerentes del sector público obtuvieron puntuaciones ligeramente más altas que sus contrapartes del sector privado, lo cual es indicativo de que pudieran estar más inclinados a la coerción que estos.

Respecto al tercer dilema, la decepción o engaño, los análisis apuntaron que no hubo diferencias estadísticamente significativas en las puntuaciones de los gerentes de los sectores público y privado de Puerto Rico. Sin embargo, se encontraron diferencias significativas entre los gerentes de Colombia y Costa Rica. De hecho, esta diferencia fue tan marcada que en el caso de Colombia, los gerentes del sector privado tuvieron una puntuación 1,81 puntos superiores que sus contrapartes del sector público. De forma similar, los gerentes costarricenses del sector privado denotaron una puntuación promedio de 1,77 puntos más alta que los gerentes del sector público de dicho país. Estos resultados refieren una tendencia muy marcada de los gerentes del sector privado en Colombia y Costa Rica por involucrarse en este tipo de comportamientos (engaño o decepción) a diferencia de los gerentes del sector público. En Puerto Rico, esta diferencia promedio fue de 0,33 puntos.

Por otra parte, con respecto a los resultados relativos al cuarto dilema sobre el robo, se identificaron diferencias estadísticamente significativas entre los gerentes de los sectores público y privado en la submuestra de gerentes puertorriqueños, pero no entre los participantes colombianos ni costarricenses. A pesar de que las puntuaciones promedio fueron muy bajas en los gerentes de ambos sectores de la economía en los tres países de referencia, pues todos los promedios fueron menores a dos puntos, en los análisis se presentan promedios más altos entre los gerentes del sector privado en Puerto Rico y Colombia. En contraste, en el caso de Costa Rica, la puntuación promedio 
fue más alta entre los participantes del sector público. Se deduce que una puntuación más alta en esta escala es más demostrativa una tendencia a tolerar el robo como una alternativa de actuación gerencial, dadas unas circunstancias específicas, tales como las expresadas en el dilema presentado en el cuestionario.

CUADRO 1

ANÁLISIS DE PRUEBA T: DIFERENCIAS DE PROMEDIOS ENTRE GERENTES DE SECTOR PÚBLICO Y PRIVADO

(EN PORCENTAJE)

\begin{tabular}{lrrrrrrrrrrrrrrr}
\hline \multicolumn{1}{c}{ Dilemas } & $\mathrm{t}$ & $\mathrm{gl}$ & Sig & med & $\mathrm{DE}$ & $\mathrm{t}$ & $\mathrm{Gl}$ & $\mathrm{sig}$ & med & DE & $\mathrm{t}$ & gl & sig & med & DE \\
\hline D1: Soborno & $-1,083$ & 104 & 0,281 & $-0,258$ &, 238 & $-0,537$ & 77 & 0,593 & $-0,222$ & 0,413 & $-1,478$ & 44,787 & 0,146 & $-0,493$ & 0,334 \\
D2: Coerción & $-1,333$ & 87,698 & 0,186 & $-0,168$ &, 126 & $-0,047$ & 77 & 0,963 & 0,019 & 0,412 & 0,790 & 94 & 0,431 & 0,102 & 0,129 \\
D3: Engaño & $-1,082$ & 102 & 0,282 & $-0,742$ &, 686 & $-2,213$ & 77 & $0,030^{*}$ & $-1,809$ & 0,817 & $-2,500$ & 63,713 & $0,015^{*}$ & $-1,770$ & 0,708 \\
D4: Robo & $-1,988$ & 95,864 & $0,050^{*}$ & $-0,961$ &, 483 & $-1,328$ & 76,99 & 0,188 & $-0,775$ & 0,583 & 0,493 & 94 & 0,623 & 0,272 & 0,551 \\
D5: Discriminación & $-0,516$ & 104 & 0,607 & $-0,296$ &, 573 & 2,006 & 54,749 & $0,050^{*}$ & 1,726 & 0,861 & $-1,301$ & 94 & 0,196 & $-0,6935$ & 0,532 \\
D6: Transparencia & $-0,411$ & 102 & 0,682 & $-0,286$ &, 695 & $-2,541$ & 77 & $0,013^{*}$ & $-1,941$ & 0,764 & $-2,169$ & 93 & $0,033^{*}$ & $-1,676$ & 0,772 \\
\hline
\end{tabular}

Nota: *Los resultados resaltados con negrillas en el cuadro corresponden a un nivel de significancia $<0.05$

Fuente: Elaboración propia

En el análisis de los resultados del quinto dilema sobre la discriminación laboral por origen nacional, no se encontraron diferencias estadísticamente significativas entre las puntuaciones de los gerentes de los sectores público y privado de Puerto Rico y Costa Rica. Sin embargo, sí se hallaron diferencias significativas en las puntuaciones de los gerentes públicos y privados de Colombia que formaron parte del estudio. Un análisis de los promedios apuntó a la existencia de promedios más altos entre los gerentes del sector privado de Puerto Rico y de Colombia en comparación con los gerentes del sector público encuestados en dichos países. Por el contrario, en la submuestra de gerentes costarricenses, el promedio de los gerentes del sector público fue más alto que el de sus contrapartes del sector privado. Debe recordarse que un promedio más alto en este dilema implicaba una mayor tendencia hacia la discriminación laboral por origen nacional de los solicitantes de empleo.

Por último, los resultados relativos al dilema de la transparencia no demostraron diferencias estadísticamente significativas entre los gerentes de los sectores público y privado de Puerto Rico, pero sí entre los de Colombia y Costa Rica. Por otra parte, un análisis de las puntuaciones promedio apuntó a la existencia de puntuaciones más altas entre los gerentes del sector privado de los tres países que formaron parte del estudio. Cabe aclarar, sin embargo, que una puntuación promedio más alta en este dilema indicaba una tendencia a ser menos transparente en la gestión.

En términos generales, los resultados tienden a validar parcialmente la hipótesis de trabajo número uno, ya que se encontraron diferencias estadísticamente significativas entre los gerentes de los sectores público y privado de algunos de los países incluidos en el estudio, en cuatro de los dilemas éticos planteados.

De forma similar, para indagar la posibilidad de diferencias significativas en el promedio de los gerentes en cada uno de los seis dilemas éticos por razón de su país de origen, se llevaron a cabo análisis más avanzados. Estos iban encaminados a someter a prueba la segunda hipótesis del estudio, la cual planteaba la existencia de diferencias estadísticamente significativas en el promedio de las puntuaciones de los participantes por razón de su país de origen. Para esto, se construyeron índices de tendencias éticas sumando las puntuaciones de los participantes de cada país en cada uno de los seis dilemas y obteniendo un promedio general de estas. Luego, se llevaron a cabo Análisis de Varianza unidireccionales para cada uno de los dilemas para determinar si había diferen- 
cias estadísticamente significativas por razón del país de origen de los participantes: Puerto Rico, Colombia y Costa Rica.

Los resultados revelaron que no existen diferencias estadísticamente significativas en los promedios de los gerentes participantes por razón de su país de origen en el primer dilema, el soborno $(F=0,940, p=0,392)$. De forma similar, tampoco se encontraron diferencias significativas en el segundo dilema, coerción $(F=0,816, p=0,443)$, ni en el tercer dilema, decepción o engaño $(F=$ $1,607, p=0,202)$. Tampoco se hallaron diferencias estadísticamente significativas entre los gerentes participantes por razón de su país de origen en los dilemas de robo $(F=1,642, p=0,195)$, discriminación $(F=1,779, p=0,171)$ y transparencia $(F=0,761, p=0,468)$. Es decir, que los investigadores no hallaron diferencias estadísticamente significativas en las tendencias éticas de los gerentes que formaron parte de la investigación por razón de su país de origen, a saber: Puerto Rico, Colombia y Costa Rica. La evidencia no apoyó la hipótesis de trabajo número dos, en la cual se planteaba la existencia de diferencias estadísticamente significativas en las tendencias éticas de los participantes del estudio por razón de su país de origen.

\section{Tipo de razonamiento ético prevalente en los gerentes de los sectores público y privado}

Otro propósito de la investigación fue determinar el tipo de razonamiento ético (deontológico, axiológico, utilitario, relativista u otro) que prevaleciente en los gerentes de los sectores público $y$ privado que participaron de este estudio internacional.

Aquí debe recordarse que mediante el análisis de contenido, las respuestas de los participantes del estudio, se clasificaron como deontológicas, si se originaban en la ética porque la situación planteada se relacionaba con algún código, reglamento, ley, estatuto, etc.; como axiológicas cuando se relacionaban con razones de conciencia, valores, creencias, así como con las normas o morales culturales; como utilitaria, si denotaban la búsqueda de beneficios para sí mismo, para su núcleo familiar, para la organización laboral o sus miembros, o si predominaba en ellas la intención de evitar un castigo o pena y relativista si las respuestas denotaban ambivalencia ética. Las explicaciones que no se adaptaban a las categorías anteriores, se agruparon bajo la categoría de "otra".

En el primer dilema sobre soborno, en el caso de los gerentes puertorriqueños predominó el tipo de razonamiento axiológico. En específico, se halló que el $67 \%$ de los gerentes del sector público y el 51,9\% de los del sector privado de este país actuarían de una manera ética, con base en sus valores, creencias y normas culturales. El tipo de razonamiento que ocupó el segundo lugar en este dilema entre los gerentes de este país, fue el utilitario con un $18,4 \%$ entre los gerentes del sector público y un 29,6\% entre los del sector privado puertorriqueño.

Resulta interesante indicar que, entre los tipos de respuesta más comunes a este dilema, los participantes colombianos utilizaron principalmente los tipos de razonamiento axiológico y deontológico. Así pues, de las respuestas de los gerentes del sector público, se dedujo que el 62,5\% utilizó un razonamiento de este tipo en este dilema, así como el $27,7 \%$ de los gerentes del sector privado. Por otra parte, el principal tipo de razonamiento utilizado por los gerentes del sector privado y el segundo tipo utilizado con más frecuencia por los gerentes del sector público colombiano fue el deontológico con un $44,7 \%$ y un $34,4 \%$ de la muestra respectivamente. Con respecto a este dilema, los resultados de la submuestra de gerentes costarricenses revelaron que los tipos de razonamiento más utilizados por los participantes fueron el axiológico, con un 72,9\% de los gerentes del sector público y un $73,0 \%$ de los del sector privado, y el utilitario con un $24,3 \%$ de los gerentes del sector privado y un $15,3 \%$ de los del sector público (ver cuadro 2). 
CUADRO 2

TIPO DE RAZONAMIENTO POR CADA UNO DE LOS PAÍSES DE ACUERDO CON

EL TIPO DE SECTOR EN A DILEMA 1: SOBORNO

(EN PORCENTAJE)

\begin{tabular}{|c|c|c|c|c|c|c|}
\hline \multirow[b]{2}{*}{$\begin{array}{c}\text { Tipos de } \\
\text { Razonamiento }\end{array}$} & \multicolumn{3}{|c|}{ Sector Público \% } & \multicolumn{3}{|c|}{ Sector Privado \% } \\
\hline & $\begin{array}{l}\text { Puerto } \\
\text { Rico }\end{array}$ & Colombia & $\begin{array}{l}\text { Costa } \\
\text { Rica }\end{array}$ & $\begin{array}{l}\text { Puerto } \\
\text { Rico }\end{array}$ & Colombia & Costa Rica \\
\hline Deontológico & 12,3 & 34,4 & 6,8 & 14,8 & 44,7 & 2,7 \\
\hline Axiológico & 67,3 & 62,5 & 72,9 & 51,9 & 27,7 & 73,0 \\
\hline Utilitario & 18,4 & 0,0 & 15,3 & 29,6 & 14,9 & 24,3 \\
\hline Relativista & 2,0 & 0,0 & 5,1 & 3,7 & 8,5 & 0,0 \\
\hline Otro & - & 3,1 & - & - & 4,3 & - \\
\hline Total & 100 & 100 & 100 & 100 & 100 & 100 \\
\hline
\end{tabular}

Nota: se destacan en negrillas los dos tipos de razonamiento con promedios más alto por país. Fuente: Elaboración propia

Entre las explicaciones más comunes ofrecidas por los participantes sobre por qué no obrarían de manera antiética en este primer dilema, relacionado con el soborno, se encontraron referencias a que incurrir en la práctica planteada en el dilema no sería ético, sería deshonesto, iría contra sus valores y principios $y$, en general, sería aceptar un soborno.

De otra parte, el análisis cualitativo de las respuestas de los participantes en el dilema de la coerción (segundo dilema), mostró que el 80,9\% de los participantes del sector público y el 81,5\% de los del sector privado puertorriqueño, respondieron desde una perspectiva axiológica a dicha pregunta. El tipo de razonamiento que ocupó el segundo lugar entre la submuestra de gerentes de dicho país caribeño fue el utilitario, en el cual se agrupó el 8,5\% de los gerentes del sector público y el 7,4\% de los del sector privado. De forma similar, el 75\% de los participantes del sector público colombiano, así como el 85,1\% de los del sector privado también denotaron un tipo de razonamiento axiológico como respuesta. A este tipo de razonamiento, le siguieron en términos porcentuales el deontológico, el cual fue utilizado por el 18,8\% de los gerentes del sector público y el utilitario, cuyo uso se encontró en el 8,5\% de la submuestra de gerentes del sector privado colombiano. En contraste, la submuestra de gerentes costarricenses demostró inclinarse principalmente por razonamientos de tipo axiológico; de hecho, el 96,6\% de los gerentes del sector público y el 94,6\% de los del sector privado utilizaron este tipo de razonamiento. Solo una minoría de participantes, equivalentes al 3,4\% de los del sector público y el 5,4\% de los del sector privado denotaron razonamientos de tipo utilitario (ver cuadro 3). En la mayoría de las narrativas de los encuestados de los tres países, prevalecieron explicaciones que tendían a indicar que la coerción era un acto que iba en contrario a la ética en general, la ética profesional, los valores, la propia conciencia y el principio de mérito. 
CUADRO 3

TIPO DE RAZONAMIENTO POR CADA UNO DE LOS PAÍSES DE ACUERDO CON EL TIPO DE SECTOR EN EL DILEMA 2: COERCIÓN

(EN PORCENTAJE)

\begin{tabular}{|c|c|c|c|c|c|c|}
\hline \multirow[b]{2}{*}{$\begin{array}{c}\text { Tipos de } \\
\text { Razonamiento }\end{array}$} & \multicolumn{3}{|c|}{ Sector Público \% } & \multicolumn{3}{|c|}{ Sector Privado \% } \\
\hline & $\begin{array}{l}\text { Puerto } \\
\text { Rico }\end{array}$ & Colombia & $\begin{array}{l}\text { Costa } \\
\text { Rica }\end{array}$ & $\begin{array}{l}\text { Puerto } \\
\text { Rico }\end{array}$ & Colombia & Costa Rica \\
\hline Deontológico & 6,4 & 18,8 & - & 5,6 & 6,4 & - \\
\hline Axiológico & 80,9 & 75,0 & 96,6 & 81,5 & 85,1 & 94,6 \\
\hline Utilitario & 8,5 & 3,1 & 3,4 & 7,4 & 8,5 & 5,4 \\
\hline Relativista & 4,2 & - & - & 5,5 & - & \\
\hline Otro & - & 3,1 & - & - & 0,0 & - \\
\hline Total & 100 & 100 & 100 & 100 & 100 & 100 \\
\hline
\end{tabular}

Nota: se destacan en negrillas los dos tipos de razonamiento con promedios más alto por país. Fuente: Elaboración propia

Respecto al tercer dilema o reactivo presentado en el cuestionario y relacionado con el dilema del engaño, los resultados revelaron que las categorías con los mayores porcentajes de participantes del sector público puertorriqueño fueron la axiológica con el 47,8\% de los participantes del sector público y el 39,2\% de los del sector privado, y la utilitaria con el 32,6\% de los gerentes públicos y el $45,1 \%$ de los gerentes del sector privado. Resulta interesante que para este dilema aumentó el porcentaje de participantes que ofrecieron una respuesta utilitaria al dilema en la submuestra puertorriqueña. En comparación, los tipos de razonamiento más frecuentes entre los colombianos en este dilema fueron el axiológico, observado en el 43,8\% de los gerentes del sector público y en el $22,7 \%$ del sector privado; el relativista, el cual estuvo presente en el $43,2 \%$ de los gerentes del sector privado; y el deontológico, el cual se halló en el 21,9\% de los participantes del sector público de dicho país. Llama la atención que los resultados de los participantes costarricenses revelaron una mayor inclinación hacia el tipo de razonamiento utilitarista, con un 54,8\% de los gerentes del sector público y un $71,0 \%$ de los del sector privado. A este tipo de razonamiento, le siguió en términos porcentuales el axiológico con el 35,5\% de los participantes del sector público y el 25,8\% de los del sector privado (ver cuadro 4).

Entre las justificaciones más utilizadas por los gerentes de ambos sectores, destaca en primer lugar, un grupo mayoritario de participantes que planteó que el llevar a cabo la conducta planteada (engaño) sería mentir, una falta de honestidad o una falta de ética. Igualmente, entre aquellos gerentes que predominó una perspectiva utilitaria, (la cual obtuvo el segundo lugar), sobresalieron narrativas que argumentaban la posibilidad de llevar a cabo el acto antiético para ayudar a la compañía y a sus empleados o porque la compañía tenía la capacidad de llevar a cabo el trabajo a pesar de su precaria situación financiera actual. 


\section{CUADRO 4 \\ TIPO DE RAZONAMIENTO POR CADA UNO DE LOS PAÍSES DE ACUERDO CON EL TIPO DE SECTOR EN EL DILEMA 3: ENGAÑO \\ (EN PORCENTAJE)}

\begin{tabular}{|c|c|c|c|c|c|c|}
\hline \multirow[b]{2}{*}{$\begin{array}{c}\text { Tipos de } \\
\text { Razonamiento }\end{array}$} & \multicolumn{3}{|c|}{ Sector Público \% } & \multicolumn{3}{|c|}{ Sector Privado \% } \\
\hline & $\begin{array}{l}\text { Puerto } \\
\text { Rico }\end{array}$ & Colombia & $\begin{array}{l}\text { Costa } \\
\text { Rica }\end{array}$ & $\begin{array}{l}\text { Puerto } \\
\text { Rico }\end{array}$ & Colombia & Costa Rica \\
\hline Deontológico & 10.9 & 21.9 & - & 2.0 & 13.6 & \\
\hline Axiológico & 47.8 & 43.8 & 35.5 & 39.2 & 22.7 & 25.8 \\
\hline Utilitario & 32.6 & 12.5 & 54.8 & 45.1 & 13.6 & 71.0 \\
\hline Relativista & 8.7 & 18.8 & 9.7 & 13.7 & 43.2 & 3.2 \\
\hline Otro & - & 3.0 & - & - & 6.9 & . \\
\hline Total & 100 & 100 & 100 & 100 & 100 & 100 \\
\hline
\end{tabular}

Nota: se destacan en negrillas los dos tipos de razonamiento con promedios más alto por país. Fuente: Elaboración propia

Respecto al cuarto dilema, el robo, los resultados relativos a la submuestra de gerentes puertorriqueños mostró un predominio de los tipos de razonamiento axiológico, con el 63,6\% de los gerentes del sector público y el 58,5\% de los del sector privado. La segunda categoría con los porcentajes más altos fue la utilitaria, con el 18,2\% de los gerentes del sector público y el 28,3\% de los del sector privado. Entre los gerentes colombianos, los tipos de razonamiento más utilizados en este dilema fueron el axiológico, con un $62,5 \%$ de los gerentes del sector público y un $66,0 \%$ del sector privado. A este, le siguió en términos porcentuales el deontológico, con un 18,8\% de los participantes del sector público y un $14,9 \%$ de los del sector privado. Por otro lado, aunque el tipo de razonamiento que predominó entre la submuestra de gerentes de Costa Rica también fue el axiológico, con el $54,2 \%$ de los gerentes del sector público y el $43,2 \%$ de los del sector privado, el tipo de razonamiento en segundo lugar en términos porcentuales fue el utilitarista con el 27,1\% de los encuestados del sector público y el 29,7 \% de los del sector privado, con lo cual asemeja sus resultados a los de Puerto Rico (ver cuadro 5). Entre las explicaciones más comunes ofrecidas por los participantes de ambos sectores indican que no sería ético o moral, que no sería correcto o justo, y que iría contra la posibilidad de ascender de puesto por méritos propios. En segundo lugar, estaban las narrativas que indicaban el miedo a perder el empleo o a ser procesado por violar alguna ley o reglamento. 
CUADRO 5

TIPO DE RAZONAMIENTO POR CADA UNO DE LOS PAÍSES DE ACUERDO CON EL TIPO DE SECTOR EN

EL DILEMA 4: ROBO

(EN PORCENTAJE)

\begin{tabular}{lrrrrrr}
\hline & \multicolumn{3}{c}{ Sector Público \% } & \multicolumn{3}{c}{ Sector Privado \% } \\
\hline $\begin{array}{c}\text { Tipos de } \\
\text { Razonamiento }\end{array}$ & $\begin{array}{c}\text { Puerto } \\
\text { Rico }\end{array}$ & Colombia & $\begin{array}{c}\text { Costa } \\
\text { Rica }\end{array}$ & $\begin{array}{c}\text { Puerto } \\
\text { Rico }\end{array}$ & Colombia & Costa Rica \\
\hline Deontológico & 11,4 & 18,8 & 3,5 & 5,7 & 14,9 & 13,5 \\
Axiológico & 63,6 & 62,5 & 54,2 & 58,5 & 66,0 & 43,2 \\
Utilitario & 18,2 & 6,3 & 27,1 & 28,3 & 8,5 & 29,7 \\
Relativista & 6,8 & 6,3 & 15,2 & 6,8 & 6,4 & 13,6 \\
Otro & - & 6,4 & - & 0,7 & 4,2 & - \\
Total & 100 & 100 & 100 & 100 & 100 & 100 \\
\hline
\end{tabular}

Nota: se destacan en negrillas los dos tipos de razonamiento con promedios más alto por país. Fuente: Elaboración propia

Más adelante, se examinó el tipo de razonamiento utilizado por los gerentes al exponérseles el quinto dilema, la discriminación (por origen étnico o nacional). Los resultados señalan que, entre los participantes puertorriqueños, predominaron los tipos de razonamiento axiológico y relativista. De hecho, el 47,8\% de los gerentes del sector público y el 43,4\% de los gerentes del sector privado de este país demostraron seguir un tipo de razonamiento axiológico. En segundo lugar, el 37,0\% de los gerentes del sector público y el 45,3\% de los gerentes del sector privado puertorriqueño utilizaron razonamientos de tipo relativista al enfrentar este dilema. Por otra parte, entre los participantes colombianos, los tipos de razonamiento más utilizados en este dilema fueron el axiológico, con un $34,4 \%$ de los participantes del sector público y el 31,9\% de los del sector privado; el deontológico, en el cual se encontró el 36,2\% de los gerentes del sector privado y el relativista, con un 31,3\%, de los gerentes del sector público. En contraste con los resultados de Puerto Rico y Colombia, entre los gerentes costarricenses encuestados, el tipo de razonamiento que predominó en este dilema fue el utilitarista, con el 76,3\% de las respuestas de los gerentes públicos y el 86,5\% de los privados. En segundo lugar, estuvieron el tipo de razonamiento relativista con un 15,3\% de los gerentes del sector público y 10,8\% de los participantes del sector privado (ver cuadro 6).

Entre las explicaciones más utilizadas por los participantes de ambos sectores, predominó que se debía contratar al solicitante de origen extranjero y con mayor preparación académica, pues es lo justo, ético y de acuerdo con el mérito. Por otra parte, un segmento de los participantes alegó que darían el trabajo a la persona originaria del país, pues además de la preparación, la experiencia y las ejecutorias profesionales, también debían valorarse el conocimiento de los contextos nacionales y las idiosincrasias de cada país (aunque en la situación se señalaba que ambos tenían excelente experiencia laboral), así como que en la entrevista de selección era que se sabría quién es el mejor candidato. Otros, abiertamente señalaban que le darían el trabajo al nacido en el país, solo por ese hecho o porque "conoce nuestra cultura y sistema". 
CUADRO 6

TIPO DE RAZONAMIENTO POR CADA UNO DE LOS PAÍSES DE ACUERDO CON EL TIPO DE SECTOR EN EL DILEMA 5: DISCRIMINACIÓN

(EN PORCENTAJE)

\begin{tabular}{lrrrrrr}
\hline \multicolumn{1}{c}{ Sector Público \% } & \multicolumn{3}{c}{ Sector Privado \% } \\
$\begin{array}{l}\text { Tipos de } \\
\text { Razonamiento }\end{array}$ & Puerto Rico & Colombia & Costa Rica & Puerto Rico & Colombia & Costa Rica \\
\hline Deontológico & 4,3 & 25,0 & 3,3 & 7,5 & 36,2 & 2,7 \\
Axiológico & 47,8 & 34,4 & 5,1 & 43,4 & 31,9 & 0,0 \\
Utilitario & 8,7 & 3,1 & 76,3 & 3,8 & 10,6 & 86,5 \\
Relativista & 37,0 & 31,3 & 15,3 & 45,3 & 21,3 & 10,8 \\
Otro & 2,2 & 6,2 & - & - & 0,0 & - \\
Total & 100 & 100 & 100 & 100 & 100 & 100 \\
\hline
\end{tabular}

Nota: se destacan en negrillas los dos tipos de razonamiento con promedios más alto por país.

Fuente: Elaboración propia

Por último, se evaluó el tipo de razonamiento utilizado por los gerentes al exponérseles el sexto dilema: la transparencia. Los resultados de la submuestra de gerentes de Puerto Rico reveló el predominio de los tipos de razonamiento axiológico, con un 42,2\% de los gerentes del sector público y un $27,1 \%$ de los del sector privado, así como del tipo de razonamiento utilitario, con el $28,9 \%$ de los gerentes del sector público y el 33,3\% de los del sector privado. Precisamente, esos dos tipos de razonamiento fueron los predominantes en la submuestra de gerentes colombianos. En esta, el 40,6\% de los gerentes del sector público y el 26,1\% de los del sector privado utilizaron un tipo de razonamiento axiológico al enfrentar el dilema de la transparencia. Por otra parte, el 32,6\% de los gerentes del sector privado y el 21,9\% de los del sector público siguieron un razonamiento utilitario. Una tendencia similar, se encontró entre los participantes costarricenses. En su caso, el 34,5\% de los participantes del sector público y el 27,0\% de los del sector privado manifestaron tipos de razonamiento axiológico. El segundo tipo de razonamiento utilizado por los gerentes de Costa Rica en este dilema fue el relativista, con 30,9\% de los gerentes públicos y el 32,4\% de los privados (véase cuadro 7).

\section{CUADRO 7}

TIPO DE RAZONAMIENTO POR CADA UNO DE LOS PAÍSES DE ACUERDO CON EL TIPO DE SECTOR EN EL DILEMA 6: TRANSPARENCIA

(EN PORCENTAJE)

\begin{tabular}{lrrrrrr}
\hline \multicolumn{1}{c}{ Sector Público \% } & \multicolumn{3}{c}{ Sector Privado \% } \\
\hline \multicolumn{1}{c}{ Tipos de } \\
Razonamiento & Puerto Rico & Colombia & Costa Rica & Puerto Rico & Colombia & Costa Rica \\
\hline Deontológico & 4,3 & 25,0 & 3,3 & 7,5 & 36,2 & 2,7 \\
Axiológico & 47,8 & 34,4 & 5,1 & 43,4 & 31,9 & 0,0 \\
Utilitario & 8,7 & 3,1 & 76,3 & 3,8 & 10,6 & 86,5 \\
Relativista & 37,0 & 31,3 & 15,3 & 45,3 & 21,3 & 10,8 \\
Otro & 2,2 & 6,2 & - & - & 0,0 & - \\
Total & 100 & 100 & 100 & 100 & 100 & 100 \\
\hline
\end{tabular}

Nota: se destacan en negrillas los dos tipos de razonamiento con promedios más alto por país.

Fuente: Elaboración propia 


\section{CONCLUSIONES}

Los resultados descriptivos de esta investigación apuntaron a que la mayor parte de los gerentes que formaron parte del estudio tomarían una decisión ética ante un escenario ético ambiguo o confuso. Tales resultados son cónsonos con los resultados de Fritzsche y Oz (2007).

Por otra parte, y relacionado con la hipótesis principal del estudio, que planteaba que habría diferencias estadísticamente significativas en las disposiciones éticas de los gerentes del sector público y los del sector privado, los resultados fueron un tanto complejos o confusos, aunque la apoyan parcialmente. En primer lugar, en los dilemas de soborno y coerción, no se hallaron diferencias estadísticamente significativas por razón del sector en el cual se desempeñaban los gerentes encuestados. Por otro lado, se encontraron diferencias significativas en el dilema de engaño en los casos de Colombia y Costa Rica, siendo los participantes del sector privado los que demostraron una mayor inclinación hacia el comportamiento antiético en este dilema. Resultados similares se encontraron para el dilema de robo en el caso de Puerto Rico, el de discriminación en el caso de Colombia, y el de transparencia en los casos de Colombia y Costa Rica.

En términos generales, se observaron unas tendencias éticas ligeramente más fuertes entre los gerentes del sector público que entre los gerentes del sector privado que formaron parte del estudio. Estos hallazgos son cónsonos con los de Roozen, Pelsmacker \& Bostyn (2001), en cuya investigación se encontró que los gerentes de organizaciones cuyo fin principal es la maximización de ganancias (sector privado) obtuvieron puntuaciones más bajas en los aspectos éticos del proceso decisorio que sus contrapartes en el sector público. De otra parte, difieren parcialmente de los de Chun (2009), en cuyo estudio se encontraron diferencias estadísticamente significativas en los valores de los empleados por razón del tipo de organización, pública o privada, a la cual pertenecían y en el cual los empleados del sector público denotaron puntuaciones más bajas en la escala de valores éticos que sus contrapartes del sector privado.

Llama la atención de los investigadores, aunque se deben considerar las limitaciones muestrales del estudio, la ausencia de diferencias estadísticamente significativas en el dilema de discriminación en los casos de Puerto Rico y Colombia, ni en el dilema de la transparencia en el caso de Puerto Rico. Siguiendo a Van Der Wal, Hubers, Den Heuvel \& Kolthoff (2006), estos son valores más ligados a la ética en el sector público que en el sector privado. Se espera que estos valores estén más aferrados entre los gerentes del sector público, pues son los encargados de poner en práctica las disposiciones de las constituciones y de otra legislación laboral contra todo tipo de discrimen, sobre todo en el ámbito laboral en sus países. Igualmente, aunque el derecho a la información de los ciudadanos en Puerto Rico se encuentra en una etapa formativa, existen varios estatutos y decisiones judiciales que dejan claro la necesidad de transparencia en los procesos gubernamentales, lo cual no necesariamente aplica al sector privado; razón por la cual hubiésemos esperado mayores diferencias entre los gerentes de los sectores público y privado en este dilema.

Por otro lado, en el estudio no se encontraron diferencias estadísticamente significativas en las disposiciones éticas promedio de los gerentes participantes por razón del país en el cual se desempeñaban, tal y como se planteaba en la segunda hipótesis del estudio. Estos resultados difieren de los de Lim (2003) y con las conclusiones generales de la investigación de Fritzsche et al. (1995), en la cual se establecía la cultura como un elemento que añadía variabilidad a las formas en las cuales los gerentes enfrentaban una situación éticamente ambigua. Debemos aclarar, sin embargo, que por haberse tomado la muestra del presente estudio en tres países latinoamericanos, quizás exista entre ellos mayor homogeneidad en el campo de los valores que la que los investigadores originalmente habían esperado. Asimismo, deben reconocerse las limitaciones que un muestreo de tipo referencial y la dificultad para reclutar gerentes de ambos sectores, le impusieron a esta investigación. Aun así, los autores puntualizan la importancia de este estudio como uno pionero en estos temas en Latinoamérica. 
Por otra parte, los resultados cualitativos del estudio revelaron que el razonamiento axiológico prevaleció entre los gerentes, tanto del sector público como del sector privado, que formaron parte del estudio al enfrentar un dilema o situación éticamente ambigua. Tales resultados divergen en este aspecto particular de los de Wheeler y Brady (1998), así como con los de Alavi, Yasin \& Zimmerer (2004), entre cuyos participantes predominó el tipo de razonamiento deontológico. Resulta interesante que, en los tres países que formaron parte del estudio, los Gobiernos han puesto gran énfasis en la aprobación de legislación y reglamentos anticorrupción. Los resultados de este estudio apuntan a la educación y al adiestramiento son mejores vehículos para promover la ética y combatir la corrupción que las medidas legales y reglamentarias. Es decir, mientras que en las organizaciones de los países estudiados, tanto las instituciones públicas como privadas han enfatizado el uso medidas de carácter deontológico para promover la ética y combatir la corrupción, la evidencia que surge de este estudio apunta a que las medidas de carácter axiológico pudieran generar mejores resultados.

De hecho, fueron muy pocas las instancias en las cuales el razonamiento de tipo deontológico se halló entre las primeras dos explicaciones que dieron los participantes en los seis dilemas éticos planteados en el estudio. Estos hallazgos deben ser tomados en cuenta por los países de la región al desarrollar planes y programas de promoción de la ética y combate a la corrupción.

A los investigadores le han llamado la atención lo que llamamos "los pequeños porcientos". Esto se refiere a un remanente de gerentes, sobre todo del sector público de los países estudiados, entre los cuales, a pesar de todos los esfuerzos que se realizan en Puerto Rico, Colombia y Costa Rica para promover la ética en el servicio público y combatir la corrupción, prevalecen tendencias antiéticas. Aún con toda la exposición pública que han tenido los casos de encarcelamiento de oficiales gubernamentales corruptos, al planteársele una situación hipotética éticamente ambigua, un segmento de los encuestados manifestó tendencias antiéticas. Nos preocupa lo que harían esos gerentes ante un ofrecimiento antiético en un contexto real.

En esa misma dirección, resultan de cuidado las respuestas de un segmento de los participantes en los dilemas de robo, discriminación laboral y transparencia. Se percibió en este grupo de participantes cierta relatividad moral, en la cual se buscaban justificaciones para acciones como el aceptar documentos robados a un competidor, negarle el empleo a un extranjero con permiso de trabajo y mejores cualificaciones que su competidor nacional, y el ocultar información a la prensa y la ciudadanía sobre la destrucción del patrimonio cultural del país. Si bien estas acciones serían reprochables entre cualquier tipo de gerente o directivo de una organización, lo son más en el caso de los gerentes del sector privado, quienes también denotaron esta tendencia minoritaria en el estudio.

Los investigadores esperan que este trabajo sirva para abrir líneas de investigación sobre este tema en Iberoamérica, así como para el desarrollo de programas de adiestramiento y formación de gerentes del sector público en materia ética.

\section{REFERENCIAS}

Alavi, J., Yasin, M. M., \& Zimmerer, T. W. (2004). An empirical investigation of the values of iranian executives: Traditional versus global market-based orientations. International Journal of Management, 21(1), 108-114. Recuperado de https://www.questia.com/library/ journal/1P3-669277271/an-empirical-investigation-of-the-values-of-iranian

Boex, J. (2013). Analyzing the role of the local public sector in achieving sustainable development: Does more spending at the local level result in better development outcomes? (Informe final julio 2013). Urban Institute Center on International Development and Governance. Recuperado de http://www.urban.org/sites/default/files/publication/23891/412881-Analyzingthe-Role-of-the-Local-Public-Sector-in-Achieving-Sustainable-Development-Does-MoreSpending-at-the-Local-Level-Result-in-Better-Development-Outcomes-.PDF 
Centro Latinoamericano de Administración para el Desarrollo. (CLAD, 2008). Carta iberoamericana de calidad en la gestión pública. Recuperado de http://old.clad.org/documentos/declaraciones/ carta-iberoamericana-de-calidad-en-la-gestion-publica/at_download/file

Centro Latinoamericano de Administración para el Desarrollo. (CLAD, 2006). Código iberoamericano de buen Gobierno. Recuperado de http://old.clad.org/documentos/ declaraciones/codigoiber.pdf/at_download/file

Chun, R. (2009). Ethical Values and Environmentalism in China: Comparing Employees from State-Owned and Private Firms. Journal of Business Ethics, 84(S3), 341-348. https://doi. org/10.1007/s10551-009-0202-1

Fritzsche, D. J., Huo, Y. P., Sugai, S., Tsai, S. D.-H., Kim, C. S., \& Becker, H. (1995). Exploring the ethical behavior of managers: A comparative study of four countries. Asia Pacific Journal of Management, 12(2), 37-61. https://doi.org/10.1007/BF01734385

Fritzsche, D., \& Oz, E. (2007). Personal Values' Influence on the Ethical Dimension of Decision Making. Journal of Business Ethics, 75(4), 335-343. https://doi.org/10.1007/s10551-006-92565

Furlong, S.R. (2003). Role and importance of regulatory policy. En J. Rabin (Ed.), Encyclopedia of Public Administration and Public Policy (Vol. 2). New York: Marcel Dekker.

Griorescu, A. (2006). Public administration role in infraestructure development. (MPRA Paper $\mathrm{N}^{\circ}$ 25101). National School of Political Studies and Public Administration. Recuperado de https:// mpra.ub.uni-muenchen.de/25101/

Khuntia, R., \& Suar, D. (2004). A Scale to Assess Ethical Leadership of Indian Private and Public Sector Managers. Journal of Business Ethics, 49(1), 13-26. https://doi.org/10.1023/ B:BUSI.0000013853.80287.da

Ortiz, A. (2016). Tendencias y tipo de razonamiento ético en una muestra de gerentes de los sectores público y privado de los Estados Unidos. Ponencia presentada en el XXI Congreso del CLAD sobre Reforma del Estado y la Administración Pública. Santiago, Chile, 8-11 de noviembre de 2016 [flashdrive].

Pandey, K. (2006). The role of administration in environmental protection: An analytical study. Journal of Environmental Research and Development, 1(1), 101-103.

Roozen, I., Pelsmacker, P., \& Bostyn, F. (2001). Ethical dimensions of decision proc e s s e s of employees. Journal of Business Ethics, 33(2), 87-99.

Soon, L. G. (2003). Differences in ethical judgement between Chinese and Singaporeans: Individual reactions to self and organizational interest considerations. Singapore Management Review, 25(2), 1-24.

Transparency International Secretariat. (2017, January 25). Índice de percepción de la corrupción 2016: Urge abordar círculo vicioso de corrupción y desigualdad. El ascenso de políticos populistas en numerosos países es una señal de alerta. Recuperado de https://www. transparency.org/news/pressrelease/indice_de_percepcion_de_la_corrupcion_2016_urge_ abordar_circulo_vicioso_de

Transparency International Secretariat. (2014, December 3). Índice de Percepción de la corrupción 2014 el crecimiento transparente en riesgo. El secreto corporativo y el lavado de dinero a escala mundial hacen aún más difícil el combate a la corrupción en economías emergentes. Recuperado de https://www.transparency.org/news/pressrelease/indice_de_percepcion_de_ la_corrupcion_2014_www.youtube.com/5-Trajectories Across Race and Gender During the 1980s and 1990s. Work and Occupations, 30(4), 440-473.

https://doi.org/10.1177/0730888403256459 
Van Der Wal, Z., Hubers, L., Den Heuvel, H.V., \& Kolthoff, E. (2006). Central values of government and business: Differences, similarities and conflicts. Public Administration Quarterly, 30(3), 314-364. Recuperado de https://www.jstor.org/stable/41288274

Vera, H., \& Pietri, L. (2009). Disposiciones y el tipo de razonamiento ético utilizado por una muestra de gerentes de los sectores público y privado del sur y oeste de Puerto Rico. Revista de Administración Pública, 41(1), 79-97. Recuperado de https://revistas.upr.edu/index.php/ap/ article/view/297

Vera, H. (2016). Tendencias y tipo de razonamiento ético en una muestra de gerentes de los sectores público y privado de Puerto Rico. Ponencia presentada en el XXI Congreso del CLAD sobre Reforma del Estado y la Administración Pública. Santiago, Chile, 8-11 de noviembre de 2016.

Wheeler, G., \& Brady, F. (1998). Do public-sector and private-sector personnel have different ethical dispositions? A study of two sites. Journal of Public Administration Research and Theory, 8(1), 93-115. https://doi.org/10.1093/oxfordjournals.jpart.a024375 